\title{
Chapter 14 \\ Distribution of Radiocesium from the Radioactive Fallout in Fruit Trees
}

\author{
Daisuke Takata
}

\begin{abstract}
We report the impact of radiocesium released by the Fukushima Daiichi nuclear power plant accident on fruit trees in the vicinity. Specifically, we discuss our findings related to the absorption and translocation of radiocesium in peach (Prunus persica L. Batsch) trees because peaches are a major crop in Fukushima Prefecture.

At the time of the nuclear accident, the majority of deciduous fruit tree species had no foliage and had not entered the flowering stage. This was also the case for peach trees, although we confirmed at harvest time that radiocesium had migrated to new plant parts. One possible explanation for this is that the presence of radiocesium in the new leaves and fruits, which was not present at the time of the nuclear accident, was affected by direct radiocesium deposition on the existing aboveground plant parts and this may have been absorbed by the tree. In the year of the accident, the root uptake of radiocesium deposited on the soil contributed very little to the overall contamination compared with absorption by the trees through the above-ground plant parts. The magnitude of radiocesium translocation from the old to new plant parts was significantly different between the year of the accident and the year after the accident. We report the findings since the Fukushima Daiichi nuclear power plant accident and speculate the pathways of radiocesium entry and release in peach trees.
\end{abstract}

Keywords Bark $\bullet$ Contamination $\bullet$ Fruit tree $\bullet$ Peach $\bullet$ Radiocesium

\footnotetext{
D. Takata $(\bowtie)$

Institute for Sustainable Agro-ecosystem Services, Graduate School of Agricultural and Life

Science, The University of Tokyo, 1-1-1 Midori-cho, Nishi-tokyo-shi, Tokyo, Japan

e-mail: takata@isas.a.u-tokyo.ac.jp
} 


\subsection{Introduction}

There are numerous reports of the effects of radionuclides on annual crops, which are related to the impact of fallout from atmospheric nuclear tests conducted since 1950s (Mitsui and Tensyo 1958), reports of the Chernobyl nuclear power plant accident aftermath (IAEA 2010), and various experiments conducted under controlled conditions involving the direct application of radionuclides to plants (Ralls et al. 1971). There are also reports related to the transfer of radionuclides from the soil to perennial fruit trees, i.e., reports that examine the transfer factors (TF) for radionuclides, but are few in number. Furthermore, tree fruits are often lumped together with fruits and vegetables, such as tomatoes and strawberries (IAEA 2010). Many investigations of fruits have dealt with the translocation of radionuclides from the soil to plant parts e.g., the study of grape leaves (Zehnder et al. 1995).

Annual peach production in Japan is approximately 170,000 tons, the majority of which is intended for fresh consumption. Given the high demand for peaches for table consumption and as gifts, they fetch relatively high prices. Peaches are among the major commodities produced in Fukushima Prefecture, where the annual peach production was valued at ten billion JPY and accounted for approximately $1 / 5$ th of the total peach production in Japan in 2010 . However, there have only been a few attempts to understand the migration of radionuclides in peach trees. Therefore, there is an urgent need to clarify the impact of radionuclides on peach trees.

Following the Fukushima Daiichi nuclear power plant accident, surveys and cultivation experiments have been conducted to investigate the migration of radiocesium in peaches and other fruit trees in Fukushima and Tokyo. Based on information derived from these investigations, the factors related to radiocesium translocation and pathways of absorption by fruit trees in the year of the nuclear accident have been reported in this paper in addition to brief descriptions of ongoing investigations.

\subsection{Pathways of Radiocesium Absorption by Fruit Trees}

There are two apparent pathways of radiocesium entry into plants, i.e., uptake of radiocesium deposited on the soil through the roots and direct absorption of radiocesium deposited on the above-ground plant parts. For direct absorption, it is necessary to distinguish the deposition of radiocesium on existing plant parts, such as branches and the main trunk that were present at the time of the Fukushima Daiichi nuclear power plant accident, from the deposition of radiocesium in new leaves, shoots, and fruit that emerged after the accident. 


\subsubsection{Uptake by Roots}

The Chernobyl nuclear power plant accident occurred when leaves had sprouted on many tree species. There has been much discussion about the direct absorption of radiocesium by the new plant parts with deposition. However, the Fukushima Daiichi nuclear power plant accident occurred when the majority of deciduous fruit trees, with the exception of plums and a few other early blooming species, had not yet flowered or produced foliage. Thus, it is expected that the pathways and levels of radiocesium absorbed by the plants in Fukushima will differ from those in Chernobyl. As expected, in the 2011 crop of plums, which had already finished flowering at the time of the accident, radiocesium was detected at levels exceeding the tentative allowable standards. These elevated levels were due to the direct airborne deposition of radiocesium on the plum flowers. However, radiocesium was also detected in fruits, leaves, and other new plant parts of fruit species such as peach and persimmon that had not produced foliage at the time of the accident. Moreover, field crops and tree species were found where the levels detected exceeded predictions based on TF using data collected before the accident. According to data collected before ploughing in May 2011, 96\% of the radiocesium found in soil at a depth of 0-5 cm below the soil surface was radioactive after the accident (Shiozawa et al. 2011). While it has been demonstrated that radiocesium is absorbed by structures on the root surfaces (Eichert et al. 1998), with the exception of shallow-rooted species such as fig, the majority of the root mass of fruit trees is found at depths $>5 \mathrm{~cm}$. Given that the radiocesium had not yet migrated to deeper soil strata during the year when the nuclear accident occurred, it is possible that there was little uptake of radiocesium through the roots.

\subsubsection{Is It Possible to Estimate the Amount of Radiocesium Absorbed from the Soil by Fruit Trees in the First Year After the Nuclear Accident?}

The presence or absence and the contribution of soil radiocesium uptake by fruit trees following the Fukushima Daiichi nuclear power plant accident was estimated using trees that were grown under conditions where there was no radionuclide fallout on the soil. Takata et al. (2012c) also conducted a different experiment where the soil surface of container-grown "Akatsuki" peach trees had been covered 1 month before the accident (Fig. 14.1). After the accident, these plants were quickly incorporated into an experiment that aimed at estimating the uptake of radiocesium from the soil.

We used eight 5-year-old "Akatsuki" peach trees, which were grown outdoors under restricted root zone conditions in containers placed in an experimental field. Each pot contained 361 of an 8:5 mixture of loamy soil and leaf mold. The soil surface of four of the trees was covered with weed control fabric since February 11, 2011. 


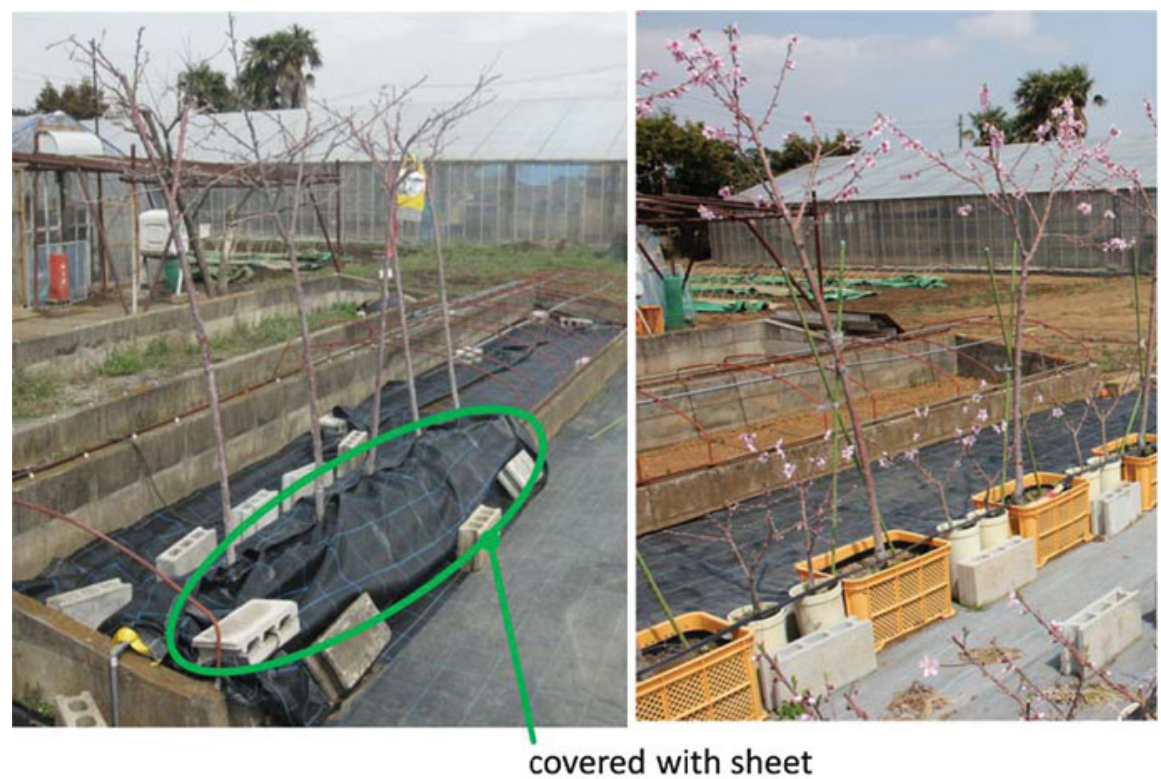

Fig. 14.1 Peach trees in containers with covering the soil surface. Left: covered (February 2011), right: without covered (March 2011)

Table 14.1 Concentration of ${ }^{134} \mathrm{Cs},{ }^{137} \mathrm{Cs}$ and ${ }^{40} \mathrm{~K}$ in soil planted "Akatsuki" peach tree in Tokyo, respectively (from Takata et al. 2012c)

\begin{tabular}{lllll}
\hline \multirow{2}{*}{ Condition } & Depth & \multicolumn{3}{l}{ Radioisotopes(Bq/kg-dry weight) } \\
\cline { 3 - 5 } Non-covered & $0-5 \mathrm{~cm}$ & $75.7 \pm 6.2$ & $87.4 \pm 7.0$ & $247.6 \pm 83.6$ \\
& $5-20 \mathrm{~cm}$ & $20.0 \pm 1.4$ & $26.7 \pm 1.7$ & $220.6 \pm 32.8$ \\
Covered & $0-5 \mathrm{~cm}$ & $15.2 \pm 1.4$ & $11.7 \pm 1.4$ & $220.4 \pm 18.0$ \\
& $5-20 \mathrm{~cm}$ & $6.7>^{\mathrm{a}}$ & $7.8>^{\mathrm{a}}$ & $228.6 \pm 13.6$ \\
\hline
\end{tabular}

andicates detection limit value

The remaining four trees, designated as the test samples, were cultivated without covering the soil surface. Peaches were harvested and the trees were dissected in July. The tree parts were separated into fruits, leaves, shoots, old branches (1-3 years old), main trunks (4 years old), and roots. Fruit were separated into the pericarp, pulp, and stones/ seeds. The old branches and main trunks were separated into the exterior parts (bark), including the cambium, and the interior parts (wood). The container soil was sampled at 0-5 and 5-20 cm. After washing, all dissected samples were oven-dried and weighed, and the amount of radiocesium in each plant part was assessed.

In the containers in which the soil was covered, the radiocesium level in the surface soil layer was approximately $1 / 7$ th of that in the uncovered soil and below the detection limit in the deeper soil (Table 14.1). We attributed the detection of radiocesium in the surface soil of the covered containers to the relatively porous cover used to cover the soil because of the low flood tolerance of peaches. We also detected radiocesium with an 
Table 14.2 Concentration of ${ }^{134} \mathrm{Cs},{ }^{137} \mathrm{Cs}$ and ${ }^{40} \mathrm{~K}$ in container grown "Akatsuki" peach tree in Tokyo, respectively (from Takata et al. 2012c)

\begin{tabular}{|c|c|c|c|c|}
\hline \multirow[b]{2}{*}{ Condition } & \multirow[b]{2}{*}{ Part } & \multicolumn{3}{|c|}{ Radioisotopes(Bq/kg-dry weight) } \\
\hline & & ${ }^{134} \mathrm{Cs}$ & ${ }^{137} \mathrm{Cs}$ & ${ }^{40} \mathrm{~K}$ \\
\hline \multirow[t]{13}{*}{ Non-covered } & Mature fruit & & & \\
\hline & - Skin & $32.3 \pm 3.8$ & $52.7 \pm 4.5$ & $556.0 \pm 32.9$ \\
\hline & - Pulp & $12.1 \pm 3.5$ & $15.7 \pm 3.2$ & $515.4 \pm 56.2$ \\
\hline & - Stone with seed & $5.5>^{\mathrm{a}}$ & $6.7 \pm 2.1$ & $331.0 \pm 26.3$ \\
\hline & Shoot & $22.0 \pm 2.9$ & $23.4 \pm 3.2$ & $415.2 \pm 50.7$ \\
\hline & Leaf & $31.5 \pm 2.9$ & $40.9 \pm 3.4$ & $908.3 \pm 93.3$ \\
\hline & Branch & & & \\
\hline & - Bark & $832.9 \pm 14.6$ & $998.6 \pm 14.1$ & $201.3 \pm 100.8$ \\
\hline & - Wood & $14.2 \pm 7.3$ & $17.4 \pm 2.5$ & $131.5 \pm 33.0$ \\
\hline & Trunk & & & \\
\hline & - Bark & $321.8 \pm 19.7$ & $389.8 \pm 11.7$ & $184.9 \pm 46.0$ \\
\hline & - Wood & $12.8 \pm 7.2$ & $15.5 \pm 6.5$ & $143.8 \pm 22.0$ \\
\hline & Root & $2.3>^{a}$ & $4.1>^{a}$ & $116.1 \pm 10.1$ \\
\hline \multirow[t]{13}{*}{ Covered } & Mature fruit & & & \\
\hline & - Skin & $37.2 \pm 5.6$ & $44.5 \pm 6.2$ & $756.4 \pm 74.3$ \\
\hline & - Pulp & $12.6 \pm 1.7$ & $14.0 \pm 1.9$ & $474.8 \pm 31.9$ \\
\hline & - Stone with seed & $6.6>^{a}$ & $9.4 \pm 2.2$ & $315.4 \pm 32.9$ \\
\hline & Shoot & $27.7 \pm 5.9$ & $37.9 \pm 7.2$ & $618.9 \pm 102.4$ \\
\hline & Leaf & $41.6 \pm 7.7$ & $54.4 \pm 9.1$ & $1148.7 \pm 117.3$ \\
\hline & Branch & & & \\
\hline & - Bark & $761.1 \pm 15.9$ & $1047.8 \pm 19.3$ & $214.1 \pm 43.3$ \\
\hline & - Wood & $12.2 \pm 3.4$ & $9.8 \pm 3.4$ & $100.7 \pm 36.2$ \\
\hline & Trunk & & & \\
\hline & - Bark & $319.8 \pm 28.9$ & $379.0 \pm 19.1$ & $180.8 \pm 21.0$ \\
\hline & - Wood & $10.7 \pm 2.8$ & $14.9 \pm 0.8$ & $144.9 \pm 16.6$ \\
\hline & Root & $2.6>^{\mathrm{a}}$ & $2.9>^{\mathrm{a}}$ & $117.3 \pm 39.5$ \\
\hline
\end{tabular}

Indicates detection limit value

unknown isotopic composition in surface soil from another part of the experimental field covered with the same fabric, which led us to believe that radiocesium could pass through the covering material, albeit in small quantities. Next, we examined the concentration of radiocesium in different plant parts (Table 14.2). Because we did not observe any differences in the radiocesium concentrations in any of the above-ground plant parts between trees grown in covered and uncovered containers, we concluded that the amount of radiocesium absorbed by the trees through the above-ground plant parts greatly exceeded that absorbed from the soil, at least in the year of the accident. In addition, we did not detect radiocesium in the roots of trees grown in covered or uncovered containers. Thus, we concluded that it was unlikely that the root uptake of radiocesium deposited on the soil surface had a significant role under natural conditions. In addition, the results clearly indicated that the majority of the radiocesium detected in trees was absorbed through the above-ground tree parts during the year of the accident. 


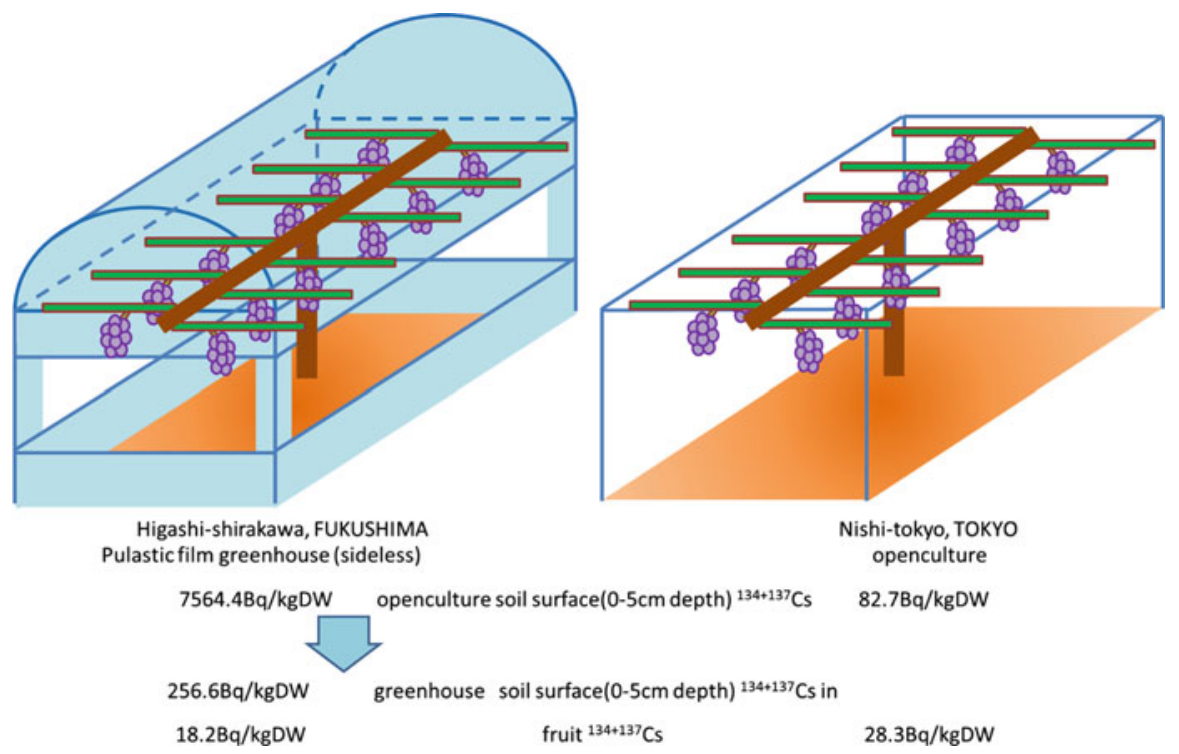

Fig. 14.2 Cultivation environment in Fukushima and Tokyo and concentration of ${ }^{134+137} \mathrm{Cs}$ in soil and grapes berry

\subsubsection{Field-Dependent Differences, After Accounting for Differences in the Cultivation Environments}

Next, we attempted to estimate the uptake of radiocesium from the soil based on the characteristics of different fields. We measured the concentration of radiocesium in various parts of "Kyoho" grape trees (Vitis spp.) cultivated in Fukushima and Tokyo. It is reasonable to assume that under the same cultivation conditions, the concentration of radiocesium in fruit would be higher in trees grown in Fukushima than those grown in Tokyo because of the higher airborne radiocesium dose rates in Fukushima. However, it would be difficult to conclude that the remaining differences in the concentration were solely attributable to differences in the soil. Therefore, we compared grapes grown in a plastic-covered greenhouse in a field in Fukushima with grapes grown in an open field in Tokyo (Fig. 14.2). In the covered field, grapes were grown in open-sided greenhouses, where the tops were covered with plastic film but the sides were open. The soil itself was not covered with plastic or fabric, but the concentration of radiocesium in the surface layer of the soil inside the greenhouse was approximately $3 \%$ of that in the adjacent uncovered areas (Fig. 14.2, bottom). The concentration of radiocesium in the surface soil was approximately three times that of the surface soil in the Tokyo field (256.8 vs 82.7$)$. The concentration of radiocesium was higher in the fruits grown in Tokyo than those grown in Fukushima. The radiocesium concentration in the other above-ground plant parts was higher for grape plants grown in the uncovered field in Tokyo or the same for grape plants grown in both fields, which did not reflect the difference in the radiocesium concentration of 
the soil. These results suggest that above-ground sources accounted for more of the radiocesium absorption by grape plants than below-ground sources at least in the year of the accident.

These experiments suggested that differences in the soil radiocesium concentration did not affect the concentration of radiocesium in various tree parts 5 months after the accident. Thus, the proportion of radiocesium absorbed by the trees through the roots was substantially lower than the proportion absorbed directly by plant parts such as the bark, which were in direct contact with radiocesium that adhered to the surface, at least in the year of the accident. Only a small proportion of radiocesium was translocated from above-ground plant parts to below-ground plant parts in the 5 months after the accident. Thus, it is difficult to estimate the absorption of radiocesium by below-ground plant parts in natural conditions, i.e., TF, at least in the year of the accident. It should be noted that the TF of plants cultivated in natural conditions are not strictly an assessment of radiocesium absorption from the soil alone, but rather represent apparent TF that also include the absorption of radiocesium deposited directly on the bark and other plant parts. Thus, in the case of radiocesium detected in the fruit of peach trees on which leaves had not sprouted at the time of the accident, there is a need to investigate the possibility, described in greater detail below, that the radiocesium was absorbed through the bark or was absorbed after being deposited by the wind or rain on leaves and fruits after sprouting.

\subsubsection{Translocation of Radiocesium from Existing Above-Ground Plant Parts}

\subsubsection{Radiocesium Concentration in the Above-Ground Plant Parts}

As explained in Sect. 14.2.1, there was a need to investigate the possibility of radiocesium absorption by the above-ground tree parts. First, we present a brief summary of an investigation that clarified the relative distribution of radiocesium in the above-ground tree parts (Takata et al. 2012b). The samples comprised three "Chiyohime" peach trees, which were grown in an experimental field (soil type: andosol) in the grounds of the University of Tokyo. On the day of harvest (June 22), we collected fruits, shoots, and leaves from bearing branches, shoots and leaves from succulent shoots, and 3-year-old branches (secondary scaffold limbs). Fruits were separated into three parts: the pericarp, pulp, and stones/seeds. In addition to harvesting the cover crop from $50 \times 50-\mathrm{cm}$ plots established approximately $75 \mathrm{~cm}$ south of the crowns of the sampled trees, we collected soil samples from the same plots at various depths. We also collected peach tree roots $(2-5 \mathrm{~mm}$ in diameter) from the same plots. We used soil samples collected from the same field on September 16, 2010, as reference samples. We also separated the succulent shoots, 3 -year-old branches, and roots into exterior parts (bark), including the cambium, and interior parts (wood). 
When the radionuclide concentrations in the shoots and fruits were low, the ${ }^{134} \mathrm{Cs}$ and ${ }^{137} \mathrm{Cs}$ concentrations in the bark of 3-year-old branches were approximately 25 times of those observed in the pulp at 759.8 and $811.6 \mathrm{~Bq} / \mathrm{kg}$ dry weight, respectively. These concentrations were also substantially higher than those in the soil and the cover crop. Thus, it is unlikely that soil-to-plant migration was the only process involved. Meteorological data for the experimental field indicated that rainfall occurred from March 21 to March 24, and it is believed that radiocesium was deposited during this period. However, the peach trees had not flowered or sprouted at this point and the flower buds still measured $3 \mathrm{~mm}$. Thus, it is difficult to imagine that the radionuclides detected in the leaves and fruits in this investigation originated solely from the intense fallout that occurred in the brief period immediately after the nuclear accident. The bark:wood radiocesium ratio varied greatly from 30:1 to 200:1 in the 3-year-old branches, particularly high radiocesium levels were detected in the bark of trees grown in Tokyo, which is a low-dose area, and Fukushima (Takata et al. 2012a, b, d, e, 2013). These results suggested that there was a need to carefully investigate the translocation of radiocesium to the bark.

There also tended to be lower partitioning of radiocesium to the fruits in highcontamination areas than in the low-contamination areas. This result suggest that, unlike potassium, which is abundant in the soil and is actively translocated to new above-ground plant parts, cesium is not translocated in large quantities or that the radiocesium was deposited directly on the leaves and fruits after sprouting.

\subsubsection{Translocation from the Bark}

We conducted an experiment using peach trees to study the inward translocation of radiocesium from above-ground plant parts, particularly to the interior of the bark layer. Using an imaging plate, we visualized the distribution of radionuclides within the main trunks of the peach trees collected in January 2012, in Date-shi, Fukushima Prefecture (Takata et al. 2012f). The images shown in Fig. 14.3 are composites of the developed imaging plates placed over regular photographs of the samples. The black spots represent areas where radionuclides were detected. It should be noted that these were 6-year-old trees; thus, the outer bark was relatively smooth and lacked the roughness that is typical of the outer bark. Furthermore, all the samples were washed before imaging. Radionuclides were detected only in the outermost layer (epidermis) of the peach tree bark and not in the layer immediately below the epidermis. Using a germanium semiconductor detector, we determined that the concentration of naturally occurring ${ }^{40} \mathrm{~K}$ did not differ in the epidermis and lower layers, implying that the different image intensities were the result of radionuclides that originated from the nuclear accident, including radiocesium. Furthermore, we used a germanium semiconductor detector to measure the radiocesium concentration in the epidermis and the layer immediately below the epidermis, which yielded the same result and showed that extremely high concentrations of radiocesium were present in the epidermis, i.e., $\geq 50$ times that in the lower layers (Takata et al. 2012d, f). Based on these observations, we considered that radiocesium deposited on the trees 

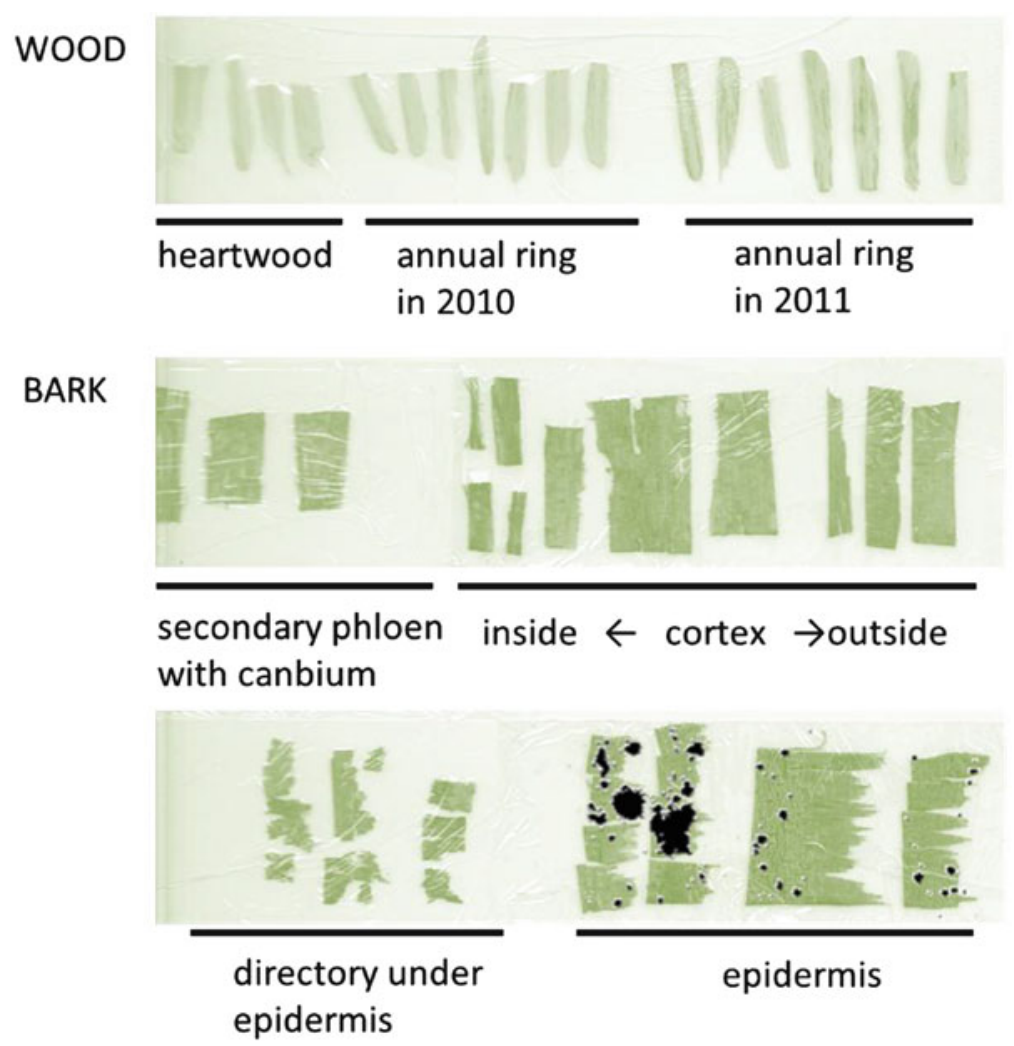

Fig. 14.3 Imaging plate of trunk in "Akatsuki" peach (Date Fukushima)

was unevenly distributed in extremely high-concentration patches within the thin outermost layer of the bark. An analogous experiment in August 2011 using imaging plates and germanium detection with peach trees collected from Higashishirakawa-gun in Fukushima Prefecture, which was a low-contamination area, produced similar results, albeit with different concentration ranges (Takata et al. 2013). However, these results do not prove that the radiocesium in the bark was translocated to internal structures such as phloem or xylem vessels. It is possible that the radiocesium was strongly bound to the epidermis itself and was not translocated in significant quantities to the water-carrying phloem located deeper in the tree. Thus, the potential and magnitude of the inward translocation of radiocesium in the epidermis is still unknown.

We can only speculate the pathways through which radiocesium migrates inward from the bark, but it is possible that it is somehow translocated through lenticels on the bark to the phloem or wood (Tanoi et al. 2012). After analyzing the imaging plates, we located radiocesium in the vicinity of lenticels (Fig. 14.4). Based on these results, we suggest that radiocesium was translocated inward through lenticels on the bark. However, lenticels are generally considered to be structures for gas 

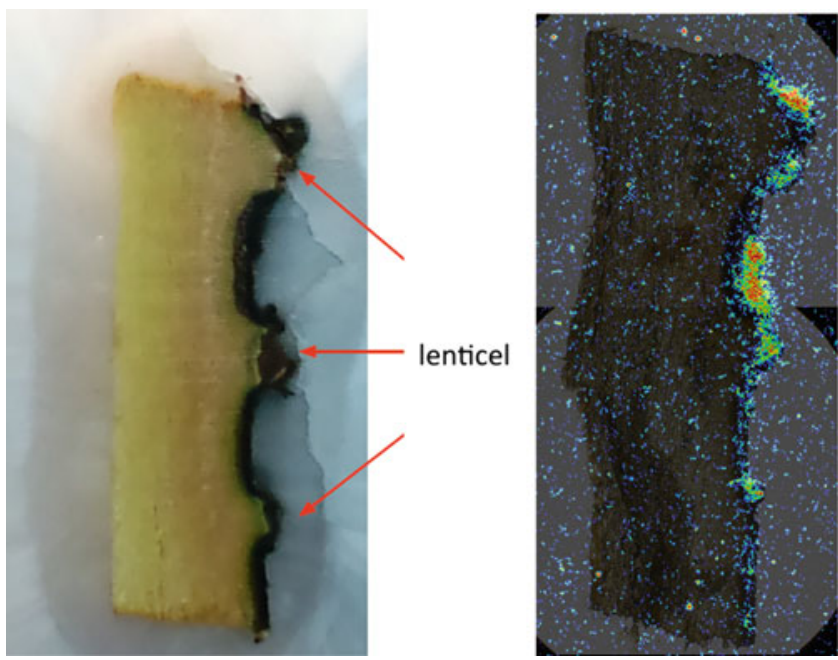

Fig. 14.4 Imaging plate of lenticel in "Akatsuki" peach (grown in Fukushima). The photo and image was obtained by Atsushi Hirose (University of Tokyo)

exchange and they exist on the bark in the form of cork. Thus, because they are relatively impermeable to water, they would not be expected to provide an effective pathway for radiocesium translocation in their typical state. Bearing this in mind, we generated imaging plates of the structures directly beneath the lenticels using peach trees collected in Fukushima as samples. We divided the lenticels into two groups - those with and without cracked centers. However, we were only able to image radionuclides in the structures below the former group of lenticels (unpublished data). The fact that radionuclides were only detected beneath cracked lenticels suggests that radionuclides were absorbed in these locations. The size of lenticels on peach tree bark varied substantially with branch age, but a greater number of lenticels with cracked centers were observed on older branches and these may be the locations where radiocesium was absorbed and translocated internally. In addition, physical injury to the branches did not only occur in the lenticels. Using imaging plates, we detected intense imaging in areas that had been scarred by pruning. In these areas, the surface was rough, which made it easier for dust accumulation, which may contain radiocesium. Thus, it is quite possible that these areas served as points for radiocesium absorption and subsequent inward translocation.

It has been reported that the concentration of radiocesium in bark decreased from summer to winter and that this was related to the sloughing away of the epidermal layer of the bark as the tree trunks expanded (Takata et al. 2012f). In ongoing experiments using washed peach trees, we have confirmed that the bark concentration of radiocesium did not change substantially between the winter and spring of 2012 (Takata et al. 2012a). This suggests that radiocesium strongly bound to the bark itself did not translocate inward during this time period. However, the concentration of radiocesium in the wood decreased from winter to spring. Reduction in the 
Table 14.3 Concentration of ${ }^{134} \mathrm{Cs}$ and ${ }^{137} \mathrm{Cs}$ in "Akatsuki" peach trees grown in Tokyo with and without washing (from Takata et al. 2012b and additional data)

\begin{tabular}{llll}
\hline & & \multicolumn{2}{l}{ Radioisotopes(Bq/kg-dry weight) } \\
\cline { 3 - 4 } Washed & Part & ${ }^{134} \mathrm{Cs}$ & ${ }^{137} \mathrm{Cs}$ \\
& Shoot & $16.3 \pm 1.2$ & $21.7 \pm 1.3$ \\
& Leaf & $76.4 \pm 11.0$ & $71.7 \pm 11.2$ \\
& Pericarp & $117.7 \pm 12.0$ & $95.9 \pm 11.7$ \\
\multirow{3}{*}{ Without washing } & Bark (3-year old branch) & $759.8 \pm 36.0$ & $811.6 \pm 36.8$ \\
& Shoot & $22.9 \pm 1.7$ & $30.8 \pm 2.9$ \\
& Leaf & $96.0 \pm 8.5$ & $107.8 \pm 10.7$ \\
& Pericarp & $133.8 \pm 18.5$ & $139.7 \pm 17.5$ \\
& Bark (3-year old branch) & $1044.6 \pm 45.8$ & $1287.8 \pm 36.8$ \\
\hline
\end{tabular}

radiocesium concentration of wood during the 2012 fruit-growth period appeared to be the result of not only dilution caused by the expansion of the wood but also the translocation of radiocesium to new plant parts. We are currently analyzing the results of these investigations (Takata et al. 2012a).

\subsubsection{Translocation from New Above-Ground Plant Parts}

Table 14.3 shows the radiocesium concentrations of the above-ground parts of washed and unwashed peach trees grown in Tokyo. The isotopic composition of radiocesium on the surface of the 3-year-old branches is unknown, but the concentration of radiocesium decreased with washing. Similarly, washing resulted in a decrease in the radiocesium concentration in the pericarps, leaves, and other new plant parts that did not exist at the time of the nuclear accident. A similar trial in Fukushima involving the measurement of radiocesium in washed and unwashed plant parts yielded similar results. It was demonstrated that irrespective of the air dose rate, it was possible to remove radiocesium from the tree surface by washing it with water. These results led to the following two speculations. First, readily mobile radiocesium continues to be present on the surface of old branches. Second, it is possible that this radiocesium was redeposited on leaves and fruits. In the case of this NPP accident, the fallout occurred only around the mid of March, 2011. However, it was possible that the radiocesium in soils or trees scattered around the fields and redeposited on leaves and fruits. It is well known that leaves take up ionic elements thorough their stomata (Eichert et al. 1998) and is reasonable to suppose that this is also the case for radiocesium. It is also possible that radiocesium was deposited by the wind and rain on the surfaces of new fruits, shoots, and leaves and was subsequently absorbed into these plant parts.

A comparison of the radiocesium concentrations in the pulp and pericarps of peaches produced in (Takata et al. 2012b) the year of the nuclear accident (2011) and 2012 (Takata et al. 2012a) showed that the ratio of the pericarp radiocesium concentration relative to the pulp radiocesium concentration was higher in 2011 than in 2012. 

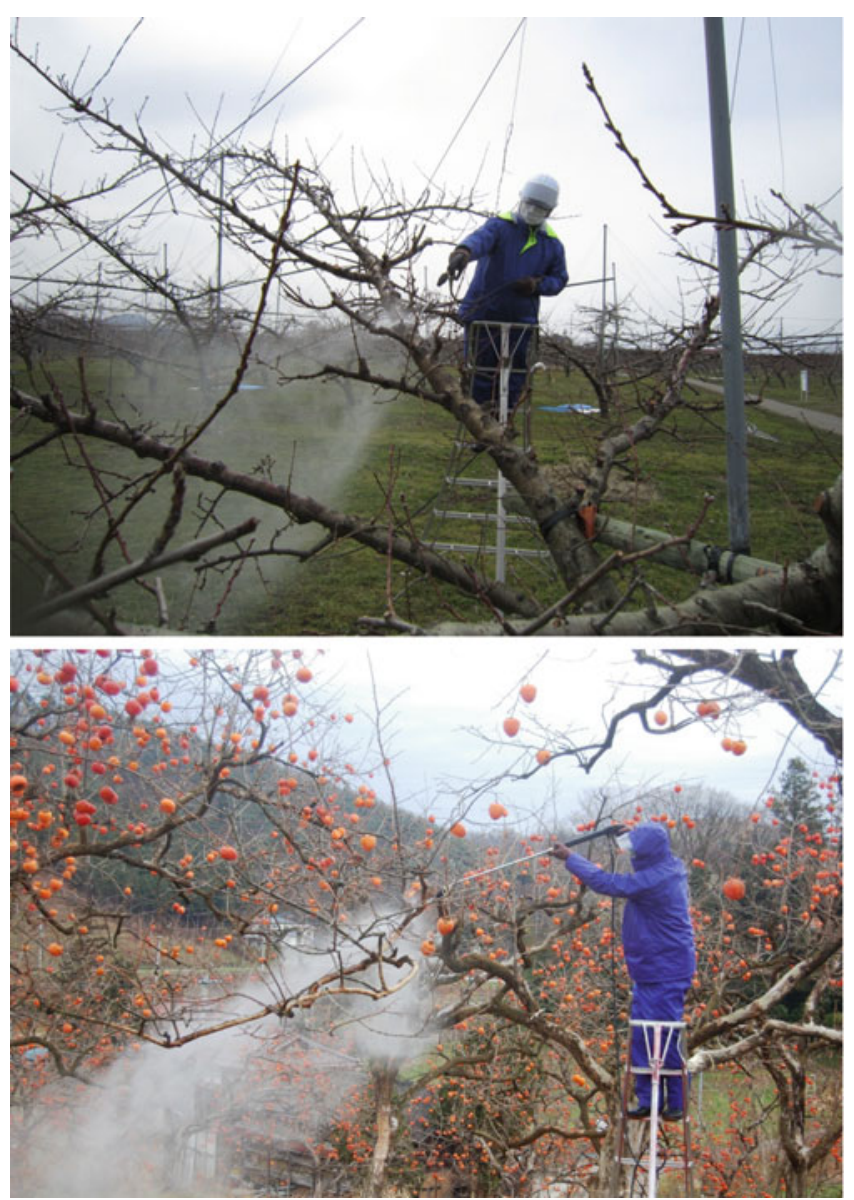

Fig. 14.5 Removing the outer layers of bark using high-pressure water sprays to peach (upper) and persimmon (bottom) trees. Photo: Mamoru Sato and Kazuhiro Abe (Fukushima Prefectural Fruit Research Center)

Based on these results, we suggest that greater quantities of radiocesium were deposited on the surfaces of fruits in 2011, thereby creating conditions where radiocesium could be more readily absorbed into the pericarp itself. Thus, it is possible that secondary deposition on new plant parts occurred for at least the first few months following the accident. A comparison of the radiocesium concentrations of the leaves growing on succulent shoots and the shoots of bearing branches (Takata et al. 2012b, 2013) showed that the radiocesium concentration in the leaves of vigorously growing succulent shoots was lower than that in the leaves of shoots on bearing branches, which ceased elongating early in the fruit growth period. There are two potential explanations for these results. First, it is possible that the redeposition of radiocesium on new leaves occurred up to the point when the shoots on the bearing branches stopped elongating (ostensibly in June). While this is difficult to verify, it is possible that the 
radiocesium existed as a readily soluble ion and that it was redeposited on leaves and fruits by rain. A second possibility is that, unlike potassium, radiocesium undergoes differential fractionation within peach trees, depending on factors such as the plant part age and growth conditions. Potassium and cesium appear to have similar behaviors in terms of root uptake (Ehlken and Kirchner 2002), but the two elements may be translocated differently in the above-ground plant parts. For example, it has been shown that cesium, unlike potassium, tends to accumulate in the older leaves in rice (Tsumura et al. 1984). Thus, with respect to the distribution of radiocesium, it was necessary to carefully examine the differences in redeposition and translocation.

In the winter of 2011, in addition to the stricter than usual enforcement of removing the outer layers of bark, farmers employed high-pressure water sprays to wash the surfaces of the peach trees in Fukushima Prefecture (Fig. 14.5). As described above, radiocesium attached to the surface can be removed by washing, which prevents it from acting as a contaminant source for redeposition onto leaves and fruits by the wind and rain. In this sense, removing the outer layer of bark and the high-pressure washing in Fukushima during the winter of 2011 were important for removing radiocesium from the tree surfaces and reducing the risk of redeposition on new plant parts in 2012.

\subsection{Release of Radiocesium from Trees}

In addition to the absorption of radiocesium by trees, we must also consider the release of radiocesium from trees. This included removal through the sloughing away of the bark, the harvesting of fruits, the falling of leaves, and release into the soil through the sloughing away or senescence of roots, as well as artificial removal by pruning. The decontamination of tree surfaces by high-pressure washing should also be included in the broader sense. To evaluate the quantity or proportion of radiocesium released or removed, it was first necessary to understand how much radiocesium was translocated to each part of the tree. We investigated the distribution of radiocesium within trees and determined various factors related to the release of radiocesium from trees based on these results.

\subsubsection{Distribution of Radiocesium Within Peach Trees 5 Months After the Nuclear Accident}

The proportion of radiocesium in fruits and leaves that were defoliated (harvested) before the winter were investigated in the experiments described below (Takata et al. 2012d). The experimental samples comprised three 5-year-old "Akatsuki" peach trees grown in an experimental field in the grounds of the University of Tokyo. Fruits were harvested on July 25, 2011, after reaching a harvestable state. The trees were subsequently excavated on August 9 and separated into leaves, shoots, 1-3-year-old branches, main trunks, rootstock, and roots. The roots were further separated according to diameter into fine roots of $<2 \mathrm{~mm}$, intermediate roots 
Table 14.4 Concentration of ${ }^{134} \mathrm{Cs}$ and ${ }^{137} \mathrm{Cs}$ in "Akatsuki" peach tree grown in Tokyo (from Takata et al. 2012d)

\begin{tabular}{|c|c|c|}
\hline \multirow[b]{2}{*}{ Position } & ${ }^{134} \mathrm{Cs}$ & ${ }^{137} \mathrm{Cs}$ \\
\hline & Bq/kg-dry weight & Bq/kg-dry weight \\
\hline \multicolumn{3}{|l|}{ Bearing branch } \\
\hline Shoot & $12.6>^{\mathrm{a}}$ & $15.4>$ \\
\hline Leaf & $42.7 \pm 4.3$ & $44.8 \pm 5.0$ \\
\hline \multicolumn{3}{|l|}{ Mature fruit } \\
\hline Pericarp & $44.2 \pm 5.4$ & $50.4 \pm 6.0$ \\
\hline Pulp & $27.0 \pm 4.4$ & $39.3 \pm 4.5$ \\
\hline Stone with seed & $6.3>$ & $7.5 \pm 2.4$ \\
\hline \multicolumn{3}{|l|}{ 3-Year-old branch } \\
\hline \multicolumn{3}{|l|}{ Succulent shoot } \\
\hline - Bark & $11.6 \pm 3.7$ & $14.4>$ \\
\hline- Wood & $9.4>$ & $10.4>$ \\
\hline - Leaf & $17.4 \pm 3.5$ & $16.3 \pm 4.0$ \\
\hline Bud(without washing) & $333.8 \pm 20.6$ & $419.1 \pm 23.6$ \\
\hline \multicolumn{3}{|l|}{ 1,2-Year-old branch } \\
\hline - Bark & $235.5 \pm 9.6$ & $284.2 \pm 11.1$ \\
\hline - Wood & $13.1 \pm 3.6$ & $19.5 \pm 4.5$ \\
\hline \multicolumn{3}{|l|}{ 3-Year-old branch } \\
\hline - Bark & $355.0 \pm 13.0$ & $434.6 \pm 15.5$ \\
\hline - Wood & $11.5 \pm 2.7$ & $12.8 \pm 3.0$ \\
\hline \multicolumn{3}{|l|}{ Trunk } \\
\hline \multicolumn{3}{|l|}{ Bark } \\
\hline - Epidermis & $245.5 \pm 16.2$ & $290.4 \pm 18.3$ \\
\hline - Directory under epidermis & $6.5 \pm 1.3$ & $4.7 \pm 1.5$ \\
\hline- Cortex & $5.8 \pm 1.8$ & $10.1 \pm 1.9$ \\
\hline - Secondary phloem with cambium & $19.4 \pm 3.2$ & $28.8 \pm 3.9$ \\
\hline \multicolumn{3}{|l|}{ Wood } \\
\hline - Sapwood & $19.7 \pm 3.3$ & $19.4 \pm 3.8$ \\
\hline - Heartwood & $7.0>$ & $8.8>$ \\
\hline
\end{tabular}

ander detection limit. Value means detection limit

of 2-10 $\mathrm{mm}$, and large roots of $10-50 \mathrm{~mm}$. In the trees used in this experiment, the 3-year-old branches corresponded to primary scaffold limbs, whereas the 1- and 2-year-old branches corresponded to secondary scaffold limbs and lateral branches. The shoots and 1-3-year-old branches were further separated into bark and wood. The bark of the main trunk was separated into four parts: the epidermis, the layer immediately below the epidermis, the cortex, and the secondary phloem and cambium. The wood was separated into two parts: the sapwood containing xylem and the heartwood with closed-off xylem. We also calculated the total radiocesium adsorption (Bq equivalent) for each plant part by multiplying the radiocesium concentration by the total dry weight of each plant part.

The radiocesium concentrations of the above-ground tree parts are presented in Table 14.4. In the old branches, the radiocesium concentration was substantially higher in the bark than in the wood. The radiocesium concentration was lower in the 


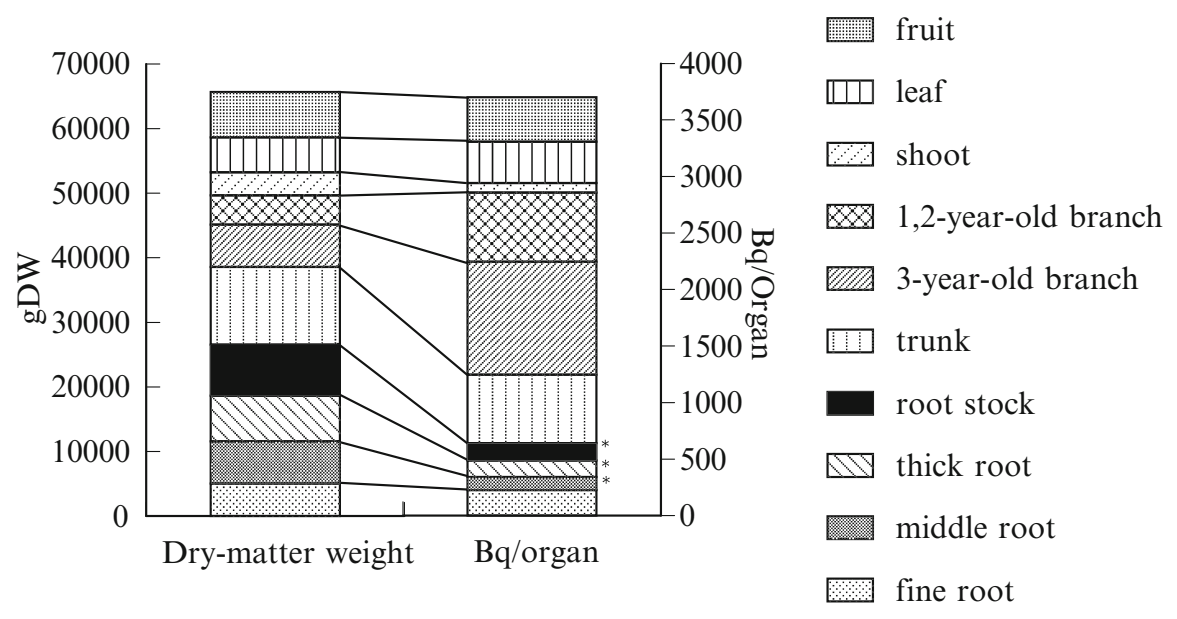

Fig. 14.6 Dry matter weight and radiocesium content for every organ in "Akatsuki" peach tree. *Under detection limit. Value means detection limit × dry weight. From Takata et al. (2012d)

main trunks than in the 3-year-old branches. Compared with the main trunks, which grew perpendicular to the ground, the 1-3-year-old branches, corresponding to the primary and secondary scaffold limbs in the sample trees, grew in a more exposed and open manner. It is possible that this difference in geometry allowed the deposited radiocesium to accumulate more readily on the surface of the 1-3-year-old branches compared with the surface of the main trunks, which accounts for the differences in the radiocesium concentration. In the bark of the main trunk, the radiocesium concentration was higher in the outermost epidermal layer than in any other part of the bark. In fact, the radiocesium concentrations in the layer immediately below the epidermis and in the cortex were only approximately $2 \%$ of that in the epidermis. It appeared that an extremely high proportion of the radiocesium was absorbed by the $0.09-0.20 \mathrm{~mm}$ thick epidermis of the main trunk. The radiocesium concentration in the secondary phloem was approximately three times that in the cortex. It is possible that this was because of absorption through the bark and translocation from leaves and other plant parts.

We calculated the total absorption of radiocesium by the different plant parts by multiplying the radiocesium concentration with the total dry weight for each plant part (Fig. 14.6). Because the radiocesium measured in all but the fine roots was below the detection limit, we tentatively assigned the detection limit to these roots. Because the detection limit was used as a proxy to calculate the total radiocesium content of the roots, the estimate represented the maximum potential concentration. Even with this upper estimate, the radiocesium content of the roots was lower than that of the above-ground parts. It was evident that the majority of the radiocesium in the peach trees was present in the above-ground parts at the time of the harvest. The radiocesium content of fruits and leaves that would not be retained until the next year accounted for approximately $20 \%$ of the total radiocesium content of the trees. 
We conducted a similar destructive sampling in Fukushima in January 2012, and we are currently analyzing the results (data unpublished). To summarize the results, despite differences in the magnitude of concentration and differences related to the presence or absence of leaves and fruits, no difference in the relative distribution of radiocesium within the trees were observed between locations. We are also in the process of determining whether it will be possible to predict the radiocesium concentration in fruits using these results.

\subsubsection{Release of Radiocesium from Above-Ground Tree Parts}

Approximately $80 \%$ of the radiocesium that was absorbed in the 5 months between the nuclear accident and the destructive sampling was expected to be retained until the next year. It has been demonstrated that the radiocesium concentration in leaves tends to decrease throughout the fruit growth period and it falls dramatically during the leaf fall period (Takata et al. 2012f). We must also consider the re-initiation of shoot elongation in the fall and the amount of radiocesium that is newly absorbed by the trees. Furthermore, with regard to the radiocesium content of branches, we must consider the removal by pruning. A study comparing peach tree pruning practices in Japan (Takata et al. 2008) showed that under conventional cultivation practices, $56.6 \%$ of peach tree branches were removed depending on the length during the winter pruning period. It is not possible to accurately calculate the mass of material removed because the above information was expressed as a ratio of the length; however, we estimated that approximately $30 \%$ of the total radiocesium content $(\mathrm{Bq}$ equivalent) of trees was removed by branch pruning. Given that the radiocesium concentration was higher in the primary and secondary scaffold limbs, which grow more horizontally than the vertically growing main trunks (Takata et al. 2012d), the active removal of secondary scaffold limbs during the winter pruning period and nurturing the succulent shoots that emerged after the nuclear accident as bearing branches in subsequent years or otherwise renewing the lateral branches, may be effective from the viewpoint of tree decontamination.

Calculating the amount of tree material lost or removed by pruning, wood expansion, and other methods, makes it possible to estimate the amount of radiocesium released by the trees over time. Examining the radiocesium concentration in peach trees in 2012 showed that in contrast to 2011, only a small percentage of the radiocesium had translocated from the old plant parts to new plant parts. Furthermore, the radiocesium concentration in the bark did not change over the fruit growth period, whereas the concentration in the wood decreased dramatically over the same time period (Takata et al. 2012f). The different distributions of radiocesium in the new plant parts in the 2 years may reflect the direct absorption of high quantities of radiocesium from the surface fruits and leaves in 2011. 


\subsubsection{The Release of Radiocesium into the Soil Through the Roots}

The peak growth of peach roots occurs in spring and fall. At the time of harvest, the radiocesium concentration of roots was low. Given that radiocesium was only present in the surface layer of the soil at the time, we assume that it would have been unlikely for deep-rooted tree species to take up significant quantities of radiocesium from the soil. Furthermore, because spring root growth utilizes nutrients stored during the previous year, we believe that there was little translocation of radiocesium from the above-ground to belowground plant parts. However, it is possible that the radiocesium accumulated in the trees was translocated to below-ground parts along with carbohydrates assimilated in the above-ground plant parts to form new roots during the fall growth. Not all the new roots of peach trees lignify and become permanent parts of the tree. A large proportion of roots senesce and are sloughed away. As roots senesce and decompose, the radiocesium contained therein is released into the soil. Thus, it is necessary to consider the transfer of radiocesium from old to new roots and the translocation of radiocesium from above-ground plant parts to below-ground plant parts.

In January 2012, we excavated peach trees grown in high dosages areas and conducted a cultivation experiment. Roots of $<2 \mathrm{~mm}$ in diameter were removed and the remaining root mass was washed. The trees were then replanted in contaminant-free soil purchased before the nuclear accident (Fig. 14.7). Although the results of this experiment are still under analysis, we have made the following observations related to the roots. The radiocesium concentration was higher in the new peach roots than the fruits and other new above-ground plant parts. Because the trees were grown in an environment where there was no soil-to-plant transfer, we can conclude that the radiocesium contained in the new roots was translocated from older plant parts. Furthermore, radiocesium was detected in the soil that was still attached to the roots. Radiocesium may be transferred to the soil through the senescence and sloughing away of new roots, or the detachment and sloughing away of the bark of older roots due to root expansion. Thus, it is possible that radiocesium was transferred to the deeper soil in loc.l orchards through the roots. However, the total mass of new roots was lower than that of the new above-ground plant parts and thus, the total amount of radiocesium translocated to the new roots was relatively small. Therefore, it is necessary to look carefully at the quantity of radiocesium that is actually translocated. Consistent with these results, an investigation of container-grown grapes (Zehnder et al. 1995) demonstrated that radiocesium applied to leaves translocated rapidly to the below-ground parts. Thus, when considering fruit trees and the orchards where they are grown, it is important to include the uptake of radiocesium from the soil and the environment and the release of radiocesium into the soil. 


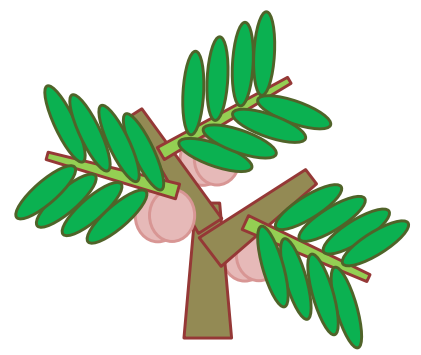

Grown in Fukushima orchard in 2011

(5-years old peach trees)

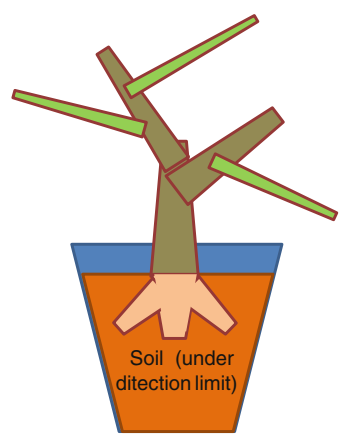

Conveyed to Tokyo and replanted

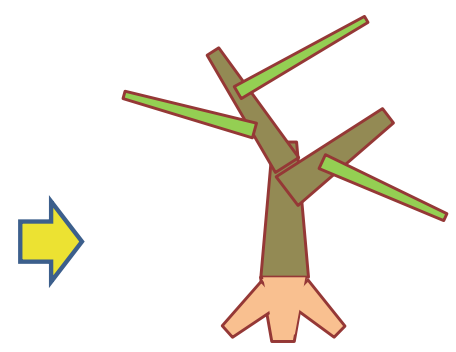

Dug, washed and removed fine root in Jan. 2012

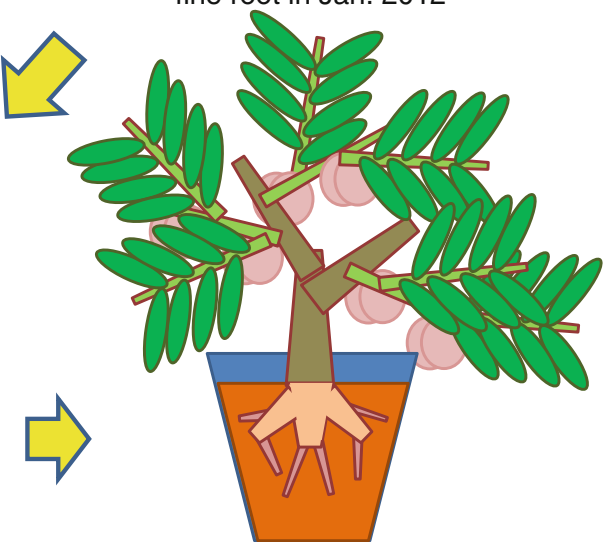

Harvest and determined

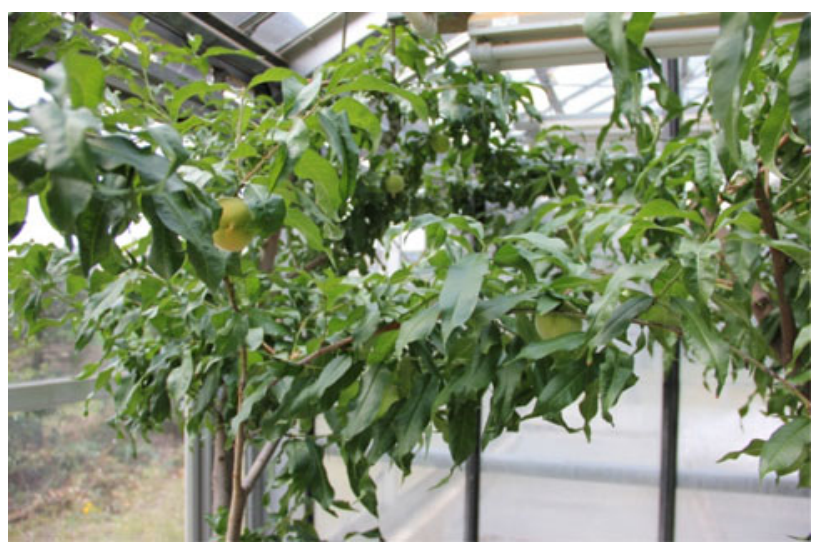

Fig. 14.7 Experimental illustration (upper) and photo (bottom) in translocation from old organ to new organ in "Akatsuki" peach trees 


\subsection{Conclusions}

Compared with other crops, there have been relatively few studies related to the migration of radiocesium in fruit trees. The same is true of experiments related to the cultivation of fruit trees. Limiting factors include the size of trees, the effort required to conduct such experiments, the duration of an experiment, considering that it may take several years for trees to become harvestable, and the difficulty in repeating experiments because of such time factors. Potted test results were sometimes different from those of orchard-based experiments. Unlike annual crops, fruit trees have wellestablished root systems; thus, the actual analysis of responses to the nuclear accident, including the felling or replanting of trees, were hindered by significant obstacles, particularly the loss of production due to the non-harvestable period following replanting. If the soil has to be decontaminated in conjunction with replanting, it is necessary to consider the amount of labor and effort required for such remediation, as well as the disposal of the contaminated soil and tree material. In the case of peach trees, it may also be unwise to replant all the trees at one time given the potential problem of replant soil sickness. Thus, for fruit trees that are perennial, it is necessary to clarify where the radiocesium absorbed in 2011 accumulated in the trees and how this radiocesium was subsequently translocated. However, this analysis also requires a certain amount of time. If the trees are not to be replanted, there is an even greater urgency for this clarification. Although radiocesium is bound strongly by the soil, it does not mean that it is not taken up at all by the roots. Furthermore, it is possible that the radiocesium released by roots, particularly that released in the vicinity of the rhizosphere of fruit trees, is reabsorbed by the trees. If no remedial action is taken, it is expected that the magnitude of radiocesium migration in the soil and the contribution of soil radiocesium to overall tree contamination will increase gradually with time.

In fruit trees and other plant species, among the potential sources of radiocesium contamination of new plant parts include soil radiocesium, radiocesium accumulated within the plants in the year of the nuclear accident, radiocesium resuspended in the surface layer of the soil, and radiocesium redeposited on new plant parts after its surface adhesion to the branches. For plants grown in contaminated soil or contaminated environments, it is difficult to determine the contribution of each of the above-mentioned sources to the radiocesium detected in new plant parts. We are currently conducting experiments using various approaches including transferring trees that were previously grown under contaminated to non-contaminated environments. We plan to report the results of these experiments in the near future.

Open Access This article is distributed under the terms of the Creative Commons Attribution Noncommercial License which permits any noncommercial use, distribution, and reproduction in any medium, provided the original author(s) and source are credited. 


\section{References}

Eichert T, Goldbach HE, Burkhardt J (1998) Evidence for the uptake of large anions through stomatal pores. Bot Acta 111:461-466

Ehlken S, Kirchner G (2002) Environmental processes affecting plant root uptake of radioactive trace elements and variability of transfer factor data: a review. J Environ Radioact 58:97-112

IAEA (2010) Handbook of parameter values for the prediction of radionuclide transfer in terrestrial and freshwater environments. Technical reports series no. 472, Austria

Mitsui S, Tensyo K (1958) Investigations on the radioactive contamination of crop plants as a result of the nuclear detonation (part 3): root uptake of fission products. J Soil Sci Plant Nutr 29:25-30 (in Japanese)

Ralls JW, Magdenberg HJ, Guckeen TR, Mercer WA (1971) Removal of radioactive strontium and cesium from vegetables and fruits during preparation for preservation. J Food Sci 36:653-656

Shiozawa S, Tanoi K, Nemoto K, Yoshida S, Nishida K, Hashimoto K, Sakurai K, Nakanishi TM, Nihei N, Ono Y (2011) Vertical concentration profiles of radioactive caesium and convective velocity in soil in a paddy field in Fukushima. Radioisotopes 60:323-328 (in Japanese with English abstract and tables)

Takata D, Fukuda F, Kubota N (2008) Effects of different cultural practice on the occurrence of reddish-pulp fruit and fruit development in 'Beni Shimizu' peach. Hort Res (Japan) 7:367-373 (in Japanese with English abstract)

Takata D, Sato M, Abe K, Yasunaga E, Tanoi K, Sasaki H, Nakanishi TM, Oshita S (2012a) Remobilization of radiocaesium derived from Fukushima nuclear power plant accident in the following year in 'Akatsuki' peach trees. Hort Res (Japan) 11(suppl 2) (in Japanese)

Takata D, Yasunaga E, Tanoi K, Nakanishi TM, Sasaki H, Oshita S (2012b) Radioactivity distribution of the fruit trees ascribable to radioactive fall out: a study on stone fruits cultivated in low level radioactivity region. Radioisotopes 61:321-326 (in Japanese with English abstract and tables)

Takata D, Yasunaga E, Tanoi K, Nakanishi TM, Sasaki H, Oshita S (2012c) Radioactivity distribution in each part of the fruit trees from radioactive fall out: transfer of radiocaesium from soil in 2011 when Fukushima Daiichi nuclear power plant accident happened. Radioisotopes 61:517-512 (in Japanese with English abstract and tables)

Takata D, Yasunaga E, Tanoi K, Nakanishi TM, Sasaki H, Oshita S (2012d) Radioactivity distribution in the fruit trees derived from radioactive fallout: radiocaesium content and its distribution in peach trees. Radioisotopes 61:607-612 (in Japanese with English abstract and tables)

Takata D, Yasunaga E, Tanoi K, Nakanishi TM, Sasaki H, Oshita S (2012e) Distribution of radioactive element derived from fallout to different parts of the peach trees. Hort Res (Japan) 11(suppl 1):279 (in Japanese)

Takata D, Yasunaga E, Tanoi K, Nakanishi TM, Oshita S, Sasaki H, Sato M, Abe K (2012f) Regional difference of radioactive cesium partitioning in peach trees contaminated by radioactive fallout. In: The 49th annual meeting on radioisotope and radiation research, p 131 (in Japanese)

Takata D, Yasunaga E, Tanoi K, Kobayashi N, Nakanishi TM, Sasaki H, Oshita S (2013) Radioactivity distribution in the fruit trees derived from radioactive fallout: a study on peach and grape cultivated in south Fukushima. Radioisotopes 61:601-607 (2012g), (Publication was rash in 2012.) (in Japanese with English abstract and tables)

Tanoi K, Hirose A, Kobayashi N, Abe K, Sato M, Nakanishi, TM (2012) Imaging analysis of radioactive cesium distribution in peach grown in Fukushima. In: International science symposium on combating radionuclide contamination in Agro-soil environment

Tsumura A, Komamura M, Kobayashi H (1984) Behavior of radioactive Sr and Cs in soils and soil-plant systems. Bull Natl Inst Agro-Environ B36:57-113 (in Japanese with English abstract)

Zehnder HJ, Kopp P, Eikenberg J, Feller U, Oertli JJ (1995) Uptake and transport of radioactive cesium and strontium into grapevines after leaf contamination. Radiat Phys Chem 46:61-69 